\title{
Modelling Conduction through the Purkinje Ventricular Junction and the Short-QT Syndrome Associated with HERG Mutation in the Rabbit Ventricles
}

\author{
OV Aslanidi, RN Sleiman, H Williamson, MR Boyett, H Zhang \\ University of Manchester, Manchester, UK
}

\begin{abstract}
Remarkable differences in action potential properties between the Purkinje fibre and ventricular cells may lead to abnormalities in excitation conduction through the Purkinje-ventricular junction (PVJ) and arrhythmogenic behaviour. We develop a family of electrophysiologically detailed computer models for rabbit epicardial, midmyocardial and endocardial ventricular myocytes, as well as the rabbit Purkinje fibre cells, in order to simulate a realistic APD dispersion during conduction through the PVJ under normal conditions and under pathological conditions of the short QT syndrome associated with HERG N588K mutation.
\end{abstract}

\section{Introduction}

Excitation conducted through the Purkinje fibre (PF) network to the ventricles determines their normal electrical activation and contraction sequence. However, remarkable differences in action potential (AP) properties between the PF and ventricular cells [1,2] may lead to abnormalities in excitation conduction through the Purkinje-ventricular junction (PVJ) and arrhythmogenic behaviour [3-5]. Primarily, as action potentials (APs) from PF cells are typically longer than ventricular APs, it has been suggested that dispersion of the action potential duration (APD) at the PVJ can result in unidirectional conduction block [3], triggered activity [4] and reentry [5] under both physiological and pathological conditions.

Computer simulations have been used previously in order to reconstruct APs in a single PF cell [6] and AP conduction in the whole fibre [7], as well as characterize electrotonic modulation of the APD dispersion at the PVJ [8]. However, the DiFrancesco-Noble model for a single rabbit PF cell [6] used in the latter simulations has been based on limited experimental data, whereas APs in ventricular cells were simulated using the Luo-Rudy model for a guinea-pig ventricular myocyte [9]. Hence, there is a demand for up-to-date non-chimeric models of the PVJ with realistic APD distributions.
The aim of this study is (i) to develop a family of electrophysiologically detailed computer models for rabbit epicardial (epi), midmyocardial (M) and endocardial (endo) ventricular myocytes, (ii) develop a detailed model for the rabbit PF cell, (iii) simulate a realistic APD dispersion due to intercellular electrotonic interactions during conduction through the PVJ under normal conditions, and (iv) explore changes of the APD dispersion under pathological conditions of the short QT syndrome (SQTS) associated with a gain-in-function of $I_{\mathrm{Kr}}$ channel due to HERG N588K mutation $[10,11]$.

\section{Methods}

Dynamics of electrical variables in cardiac tissues can be described by the Hodgkin-Huxley-type nonlinear partial differential equation (PDE) $[7,8]$ :

$$
\frac{\partial V}{\partial t}=\nabla(\mathbf{D} \nabla V)-C_{m}^{-1} I_{i o n}
$$

Here $V(\mathrm{mV})$ is the membrane potential, $t$ is time (s), $\nabla$ is a spatial gradient operator defined within the tissue geometry. D is the effective diffusion coefficient $\left(\mathrm{mm}^{2}\right.$ $\mathrm{ms}^{-1}$ ) that characterizes electrotonic spread of voltage via gap junctions. $C_{\mathrm{m}}(\mathrm{pF})$ is the cell membrane capacitance, $I_{\text {ion }}$ is the total membrane ionic current $(\mathrm{pA})$. Various biophysically detailed mathematical models have been developed to describe the voltage and time dependent current $I_{\text {ion }}$, and hence, action potential (AP) properties primarily in a rabbit ventricular cell [12].

The equation (1) is solved for the 1D strand geometry using a finite-difference PDE solver that implements the explicit Euler's method with time and space steps $\Delta t=$ $0.005 \mathrm{~ms}$ and $\Delta x=0.1 \mathrm{~mm}$, respectively.

\subsection{Ventricular models}

The LabHEART model [12] of the rabbit ventricular AP was modified to incorporate newly available experimental data on the membrane ionic currents and 


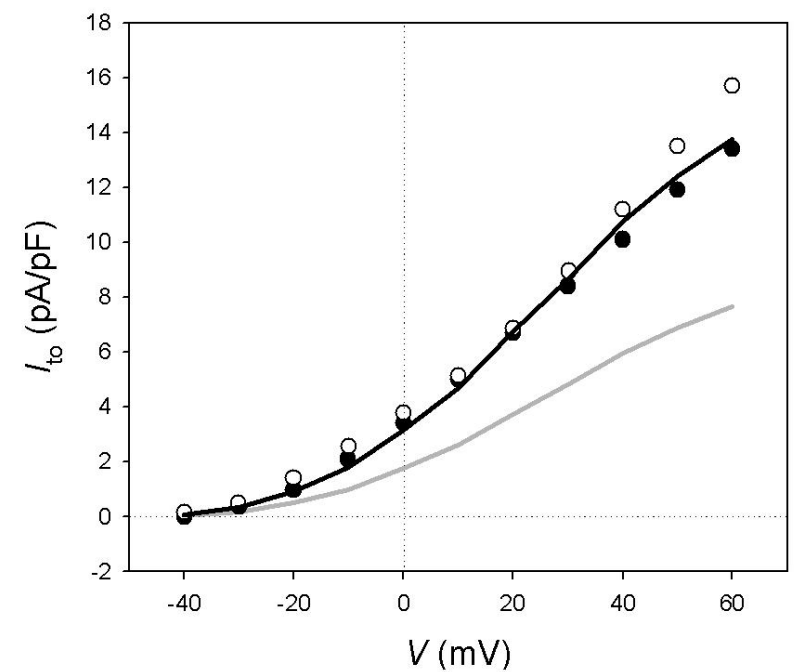

Fig. 1. Current-voltage relationships for transient outward current, $I_{\mathrm{to}}$, in a rabbit epi cell measured using the original LabHeart model (grey line), our modified model (black line) and in experiments (circles: $\circ[15], \bullet[16])$.

transmural heterogeneity of the rabbit ventricles [13-21]. Densities and activation/inactivation kinetics of all the ionic currents were modified to account for the respective experimental data: fast sodium, $I_{\mathrm{Na}}$ [13], L-type calcium, $I_{\mathrm{CaL}}[14,15]$, transient outward, $I_{\mathrm{to}}$, and outward rectifier, $I_{\mathrm{K} 1}[15,16]$, slow delayed rectifier, $I_{\mathrm{Ks}}$, and rapid delayed rectifier, $I_{\mathrm{Kr}}[15,17,18]$ and calcium activated chloride current, $I_{\mathrm{Cl}(\mathrm{Ca})}[19,20]$. Transmural differences between the epi, $\mathrm{M}$, and endo cells were introduced based on the experimentally reported differences in the current densities of $I_{\mathrm{to}}, I_{\mathrm{K} 1}[16,21], I_{\mathrm{Ks}}[17]$ and $I_{\mathrm{Cl}(\mathrm{Ca})}[20]$ in these three cell-types. Correspondence between the resultant model and the experimental data is illustrated in Fig. 1, with $I_{\text {to }}$ used as an example.

Three resultant single-cell models were incorporated into a 1D tissue model (1) with the diffusion coefficient D adopted to reproduce the transmural conduction velocity of $\sim 0.3 \mathrm{~m} / \mathrm{s}[22,23]$. Figs. 2 and 3 shows that the models also reproduce experimentally observed APs, APD restitution curves $[21,24]$ and the transmural APD dispersion patterns $[24,25]$. Note that different patterns of the APD dispersion can be simulated by changing relative sizes of the epi, $\mathrm{M}$ and endo regions.

\subsection{PF cell model}

Analogous techniques were used to construct an AP model for the rabbit PF cell. Densities and kinetics of several ionic currents were modified to account for the available experimental data: late sodium, $I_{\text {Nal }}$ [26], L-type calcium, $I_{\text {CaL }}$ [27], transient outward, $I_{\mathrm{to}}$, and outward rectifier, $I_{\mathrm{K} 1}$ [28], slow and rapid delayed rectifiers, $I_{\mathrm{Ks}}$ and $I_{\mathrm{Kr}}$ [1]. Two pacemaking current absent in the ventricular cells were added: T-type calcium, $I_{\mathrm{CaT}}$ [27], and hyperpolarization-activated "funny" current, $I_{\mathrm{f}}[29]$. Note that experimental data for $I_{\mathrm{CaL}}$ and $I_{\mathrm{CaT}}$ were recorded from canine PF cells [27] - however, we used them in our rabbit model as the AP waveforms in canine and rabbit $P F$ cells are quite similar [1]

Finally, the PF cell model was incorporated into the heterogeneous 1D strand model (1), with the non-uniform diffusion coefficient $\mathbf{D}$ adopted to produce the AP conduction velocities of $1.5 \mathrm{~m} / \mathrm{s}$ and $0.3 \mathrm{~m} / \mathrm{s}$ for the $\mathrm{PF}$ and the ventricular tissue, respectively; the values are consistent with experimental measurements [22, 23].
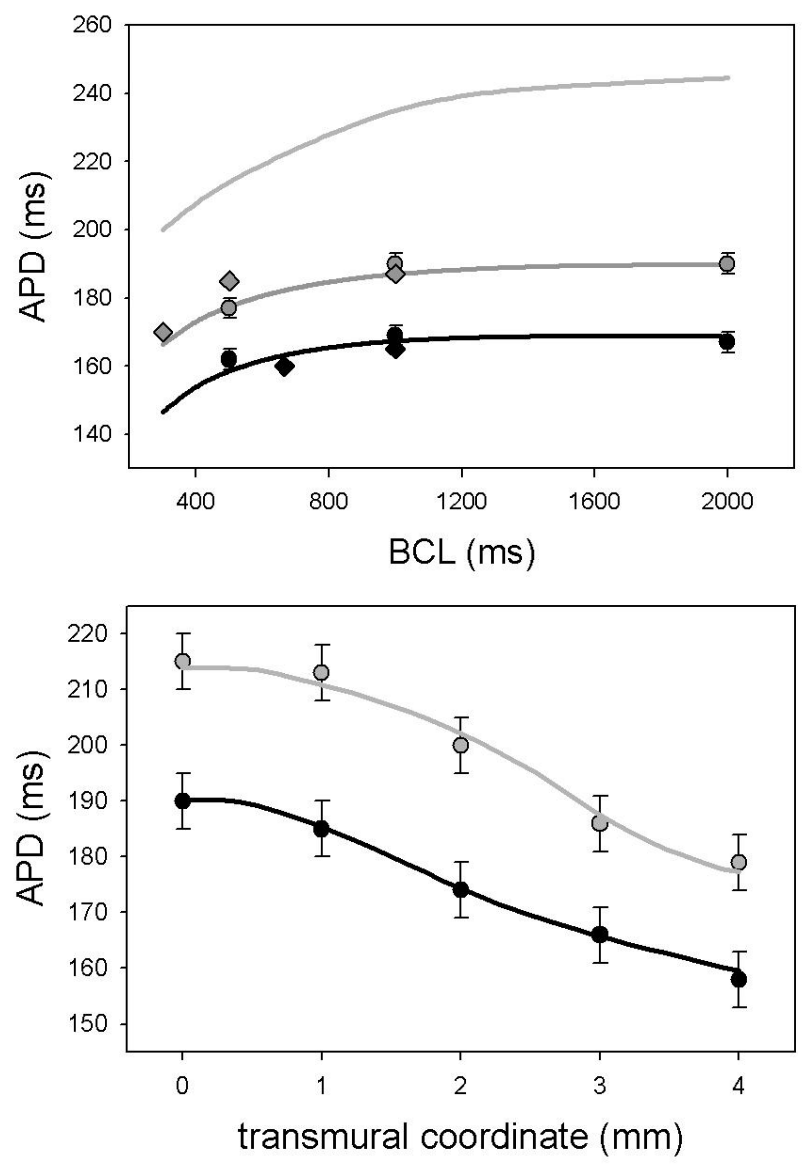

Fig. 2. Validation of the ventricular cell and tissue models. Top: the APD restitution curves simulated with the models for single epi (black line), endo (dark grey line) and M (light grey line) cells, as compared to the respective experimental measurements (diamonds [21] and circles [24]). Bottom: spatial APD dispersion patterns (lines) resultant from the transmural AP propagation in the $1 \mathrm{D}$ ventricular strand model, as compared to the respective experimental measurements $(\bullet[24], \bullet[25])$. 


\section{Results}

Simulations of the full 1D strand model produced feasible AP waveforms (Fig. 3) and the APD dispersion patterns (Fig. 4) similar to those observed experimentally, with the APD at the PVJ shorter than in the free-running $\mathrm{PF}$, but still longer than in the ventricular tissue [1-4].

Removing voltage-dependent inactivation of the delayed rectified current, $I_{\mathrm{Kr}}$, was utilized to simulate SQTS associated with a gain-in-function of $I_{\mathrm{Kr}}$ channel due to HERG N588K mutation $[10,11]$. Such "mutant" conditions enhanced net $I_{\mathrm{Kr}}$ and resulted in heterogeneous APD shortening among epi, M, endo and PF cells, which augmented the APD dispersion and shortened QT interval in the resultant pseudo-ECG (Fig. 4). This provides the first causative link between the HERG N588K mutation and SQTS. Although under the mutant conditions the APD in the PF cells was almost twice longer than in adjacent ventricular cells (Fig. 4), the PF cells were not able to drive the ventricular cells repetitively [4], which shows the importance of intercellular electrotonic interactions in modulating heterogeneity of the PVJ.

\section{Discussion and conclusions}

Our computer models reconstruct realistic APs and APD dispersion patterns within/around the rabbit PVJ under physiological and pathological conditions. Such models can be useful in clinical applications, e.g. for assessing effects of anti-arrhythmic drugs on the PVJ conduction. For instance, quinidine provides an anti-arrhythmic effect in SQTS by decreasing the APD dispersion [30] - but at the same time increases the PVJ conduction time delay [2], which may increase probability of the uni- directional conduction block [3]. Further simulations are required to study the interaction between such opposing phenomena.

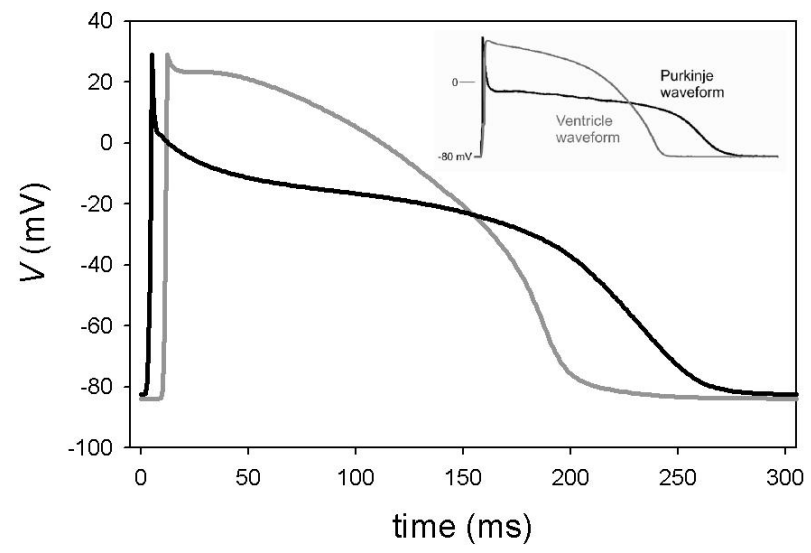

Fig. 3. APs simulated with the PF and ventricular cell models (black and grey lines, respectively), as compared to the respective experimental recordings [1].
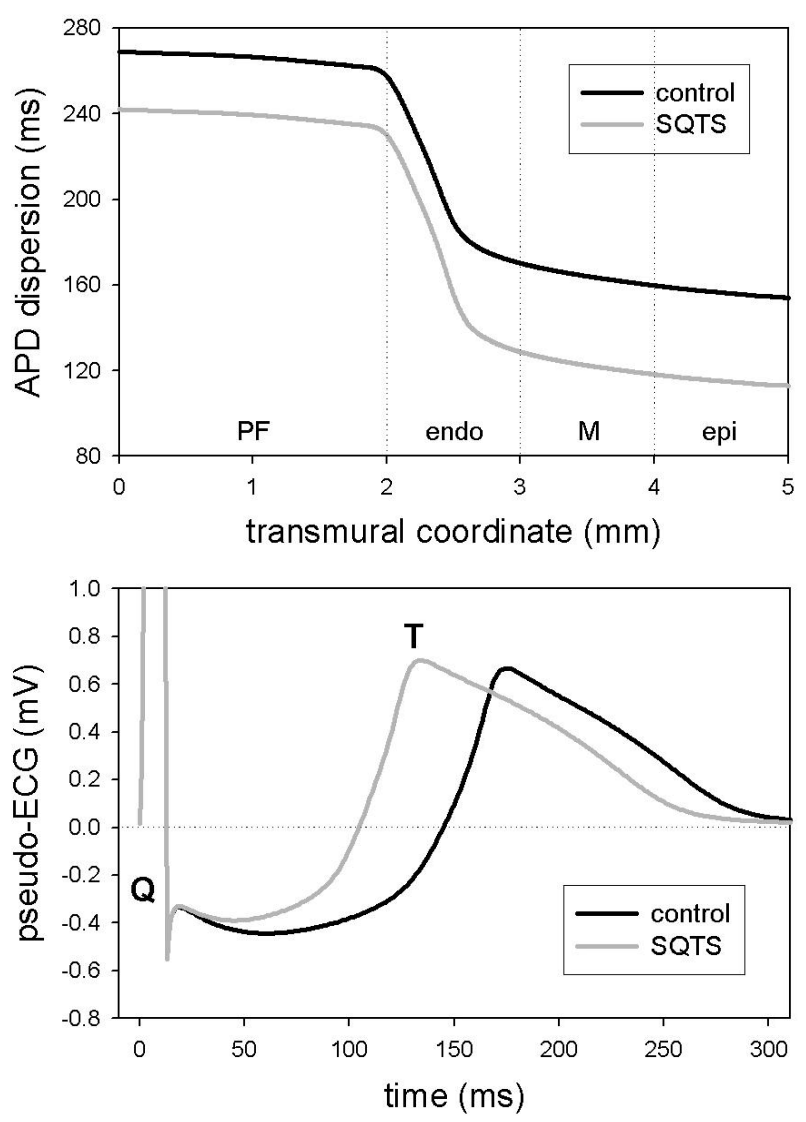

Fig. 4. SQTS in the 1D model of the PVJ conduction. Top: spatial APD dispersion patterns resultant from the AP propagation through the PVJ. Bottom: respective preuso-ECGs recorded from the epicardial surface.

Future development of the model should also take into account complex 3D morphology of the PVJ [31] in order to explore interactions between electrophysiology and anatomy of the tissue during the AP conduction.

In summary, the constructed electrophysiologically detailed models of the rabbit PF and ventricular cells and tissue provide a powerful computational tool for noninvasive studies of electrical phenomena within the heterogeneous PVJ. Shortening of the QT interval under the defected $I_{\mathrm{Kr}}$ inactivation conditions substantiates a link between SQTS and the HERG N588K mutation.

\section{Acknowledgements}

This research was supported by the BBSRC (UK).

\section{References}

[1] Dumaine R, Cordeiro JM. Comparison of $\mathrm{K}^{+}$currents in cardiac Purkinje cells isolated from rabbit and dog. J Mol Cell Cardiol 2007; 42: 378-89. 
[2] Veenstra RD, Joyner RW, Rawling DA. Purkinje and ventricular activation sequences of canine papillary muscle. Effects of quinidine and calcium on the Purkinjeventricular conduction delay. Circ Res 1984; 54: 500-15.

[3] Overholt ED, Joyner RW, Veenstra RD et al. Unidirectional block between Purkinje and ventricular layers of papillary muscles. Am J Physiol 1984; 247: H584-95.

[4] Li ZY, Wang YH, Maldonado C et al. Role of junctional zone cells between Purkinje fibres and ventricular muscle in arrhythmogenesis. Cardiovasc Res 1994; 28: 1277-84.

[5] Morley GE, Danik SB, Bernstein S et al. Reduced intercellular coupling leads to paradoxical propagation across the Purkinje-ventricular junction and aberrant myocardial activation. Proc Natl Acad Sci USA 2005; 102: 4126-29.

[6] DiFrancesco D, Noble D. A model of cardiac electrical activity incorporating ionic pumps and concentration changes. Philos Trans R Soc Lond B 1985; 307: 353-98.

[7] Cabo C, Barr RC. Reflection after delayed excitation in a computer model of a single Purkinje fiber. Circ Res 1992; 71: $260-70$.

[8] Huelsing DJ, Spitzer KW, Cordeiro JM et al. Conduction between isolated rabbit Purkinje and ventricular myocytes coupled by a variable resistance. Am J Physiol 1998; 274: H1163-73.

[9] Luo CH, Rudy Y. A dynamic model of the cardiac ventricular action potential. I. Simulations of ionic currents and concentration changes. Circ Res 1994; 74: 1071-96.

[10] Brugada R, Hong K, Dumaine R et al. Sudden death associated with short-QT syndrome linked to mutations in HERG. Circulation 2004; 109: 30-35.

[11] Zhang H, Hancox JC. In silico study of action potential and QT interval shortening due to loss of inactivation of the cardiac rapid delayed rectifier potassium current. Biochem Biophys Res Comm 2004; 322: 693-99.

[12] Puglisi JL, Bers DM. LabHEART: an interactive computer model of rabbit ventricular myocyte ion channels and $\mathrm{Ca}^{2+}$ transport. Am J Physiol 2001; 281: C2049-60.

[13] Lee HC, Matsuda JJ, Reynertson SI et al. Reversal of lidocaine effects on sodium currents by isoproterenol in rabbit hearts and heart cells. J Clin Invest 1993; 91: 693701.

[14] Rozanski GJ, Xu Z, Whitney RT et al. Electrophysiology of rabbit ventricular myocytes following sustained rapid ventricular pacing. J Mol Cell Cardiol 1997; 29: 721-32.

[15] Tsuji Y, Opthof T, Kamiya K et al. Pacing-induced heart failure causes a reduction of delayed rectifier potassium currents along with decreases in calcium and transient outward currents in rabbit ventricle. Cardiovasc Res 2000; 48: 300-09.

[16] Fedida D, Giles WR. Regional variations in action potentials and transient outward current in myocytes isolated from rabbit left ventricle. J Physiol 1991; 442: 191-209.

[17] Xu X, Rials SJ, Wu Y et al. Left ventricular hypertrophy decreases slowly but not rapidly activating delayed rectifier potassium currents of epicardial and endocardial myocytes in rabbits. Circulation 2001; 103: 1585-90.

[18] Lu Z, Kamiya K, Opthof T et al. Density and kinetics of $I_{\mathrm{Kr}}$ and $I_{\mathrm{Ks}}$ in guinea pig and rabbit ventricular myocytes explain different efficacy of $I_{\mathrm{Ks}}$ blockade at high heart rate in guinea pig and rabbit: implications for arrhythmogenesis in humans. Circulation 2001; 104: 951-56.

[19] Hirayama Y, Kuruma A, Hiraoka M et al. $\mathrm{Ca}^{2+}$-activated $\mathrm{Cl}^{-}$current is enhanced by acidosis and contributes to the shortening of action potential duration in rabbit ventricular myocytes. Jpn J Physiol 2002; 52: 293-300.

[20] Verkerk AO, Tan HL, Ravesloot JH. $\mathrm{Ca}^{2+}$-activated $\mathrm{Cl}^{-}$ current reduces transmural electrical heterogeneity within the rabbit left ventricle. Acta Physiol Scand 2004; 180: 239-47.

[21] McIntosh MA, Cobbe SM, Kane KA et al. Action potential prolongation and potassium currents in left-ventricular myocytes isolated from hypertrophied rabbit hearts. J Mol Cell Cardiol 1998; 30: 43-53.

[22] Wiedmann RT, Tan RC, Joyner RW et al. Discontinuous conduction at Purkinje-ventricular muscle junction. Am J Physiol 1996; 271: H1507-16.

[23] Sung D, Mills RW, Schettler J et al. Ventricular filling slows epicardial conduction and increases action potential duration in an optical mapping study of the isolated rabbit heart. J Cardiovasc Electrophysiol 2003; 14: 739-49.

[24] Idriss SF, Wolf PD. Transmural action potential repolarization heterogeneity develops postnatally in the rabbit. J Cardiovasc Electrophysiol 2004; 15: 795-801.

[25] Yan GX, Rials SJ, Wu Y et al. Ventricular hypertrophy amplifies transmural repolarization dispersion and induces early afterdepolarization. Am J Physiol 2001; 281: H196875.

[26] Carmeliet E. Slow inactivation of sodium current and voltage-dependent block by tetrodotoxin in rabbit cardiac Purkinje fibers. Biomed Biochim Acta 1986; 45: S163-66.

[27] Han W, Chartier D, Li D et al. Ionic remodeling of cardiac Purkinje cells by congestive heart failure. Circulation 2001; 104: 2095-100.

[28] Cordeiro JM, Spitzer KW, Giles WR. Repolarizing K ${ }^{+}$ currents in rabbit heart Purkinje cells. J Physiol 1998; 508: 811-23.

[29] Van Bogaert PP, Pittoors F. Use-dependent blockade of cardiac pacemaker current $\left(I_{\mathrm{f}}\right)$ by cilobradine and zatebradine. Eur J Pharmacol 2003; 478: 161-71.

[30] Kaufman ES. Quinidine in short QT syndrome: an old drug for a new disease. J Cardiovasc Electrophysiol 2007; 18: 665-66.

[31] Tranum-Jensen J, Wilde AA, Vermeulen JT et al. Morphology of electrophysiologically identified junctions between Purkinje fibers and ventricular muscle in rabbit and pig hearts. Circ Res 1991; 69: 429-37.

\section{Address for correspondence}

Oleg Aslanidi, Ph.D.

School of Physics and Astronomy

University of Manchester

Manchester M60 1QD

United Kingdom

oleg.aslanidi@manchester.ac.uk 